\title{
Adomian Decomposition Method Combined with Padé Approximation and Laplace Transform for Solving a Model of HIV Infection of $\mathrm{CD4}^{+} \mathrm{T}$ Cells
}

\author{
Fang Chen ${ }^{1}$ and Qing-Quan Liu ${ }^{2}$ \\ ${ }^{1}$ School of Applied Science, Beijing Information Science and Technology University, Beijing 100192, China \\ ${ }^{2}$ Key Laboratory for Mechanics in Fluid Solid Coupling Systems, Institute of Mechanics, Chinese Academy of Sciences, \\ Beijing 100190, China \\ Correspondence should be addressed to Qing-Quan Liu; qqliu@imech.ac.cn
}

Received 13 July 2015; Revised 3 September 2015; Accepted 13 September 2015

Academic Editor: Pasquale Candito

Copyright (C) 2015 F. Chen and Q.-Q. Liu. This is an open access article distributed under the Creative Commons Attribution License, which permits unrestricted use, distribution, and reproduction in any medium, provided the original work is properly cited.

\begin{abstract}
The classical Adomian decomposition method (ADM) is implemented to solve a model of HIV infection of CD4 ${ }^{+} \mathrm{T}$ cells. The results indicate that the approximate solution by using the ADM is the same as that by using the Laplace ADM, but it can be obtained in a more efficient way. We also use Padé approximation and Laplace transform as a posttreatment technique to obtain the result of the ADM. The advantage of the posttreatment is illustrated by numerical experiments.
\end{abstract}

\section{Introduce}

Because of human immunodeficiency virus (HIV), more and more people are infected with more and more casualties especially in Africa. $\mathrm{CD} 4^{+} \mathrm{T}$ cells are the most abundant white blood cells of the immune system in body. Though HIV infects also other cells, it seriously damages the $\mathrm{CD} 4^{+} \mathrm{T}$ cells in blood and this decreases the immune ability. Mathematical modeling is an important tool established for understanding HIV infection concerning $\mathrm{CD}^{+}{ }^{+} \mathrm{T}$ cells. In 1989, Perelson established a simple and important model in the field of HIV infection [1]. In 1993, Perelson et al. proposed a fitted model based on an old model which appeared in [2]. This HIV infection model of $\mathrm{CD} 4^{+} \mathrm{T}$ cells is given by the system of nonlinear differential equations:

$$
\begin{aligned}
\frac{d T}{d t} & =p-\alpha T+r T\left(1-\frac{T+I}{T_{\max }}\right)-k V T, \\
\frac{d I}{d t} & =k V T-\beta I, \\
\frac{d V}{d t} & =N \beta I-\gamma V,
\end{aligned}
$$

with the initial conditions

$$
\begin{aligned}
T(0) & =r_{1}, \\
I(0) & =r_{2}, \\
V(0) & =r_{3} .
\end{aligned}
$$

This model neglects the proliferation of infected $\mathrm{CD} 4^{+} \mathrm{T}$ cells. Here $T(t), I(t)$, and $V(t)$ describe the concentration of susceptible $\mathrm{CD} 4^{+} \mathrm{T}$ cells, $\mathrm{CD} 4^{+} \mathrm{T}$ cells infected by the HIV, and free HIV particles in the blood, respectively; $\alpha, \beta$, and $\gamma$ represent natural turnover rates of uninfected T cells, infected T cells, and virus particles, respectively; $k(>0)$ is the infection rate; $p$ denotes a rate at which the body produces $\mathrm{CD} 4^{+} \mathrm{T}$ cells from precursors in the bone narrow and thymus; $r$ represents a rate at which $\mathrm{T}$ cells multiply through mitosis when the $\mathrm{T}$ cells are stimulated by antigen or mitogen; $N$ means the virus particles that each infected $\mathrm{CD} 4^{+} \mathrm{T}$ cell produces during its life, including all its daughter cells; $T_{\max }$ indicates the maximum $\mathrm{CD}^{+}{ }^{+} \mathrm{T}$ cells concentration in the body. Also, $(1-$ $\left.(T+I) / T_{\max }\right)$ describes the logistic growth of healthy $\mathrm{CD} 4^{+} \mathrm{T}$ cells. In this paper, all parameters are the same as those in $[3,4]$; that is, $p=0.1, \alpha=0.02, \beta=0.3, r=3, \gamma=2.4$, 
$k=0.0027, T_{\max }=1500, N=10, r_{1}=0.1, r_{2}=0$, and $r_{3}=$ 0.1 .

In the recent twenty years, all kinds of models of HIV infection of $\mathrm{CD} 4^{+} \mathrm{T}$ cells have been established and studied. These models are usually described by the nonlinear differential equations. When the analytic solution can not be obtained in close form, the numerical and/or approximate solutions need to be computed. Adomian decomposition method (ADM) [5] and Laplace ADM (LADM) [6] are some typical approaches, which give the approximate solution of linear or nonlinear problems [7]. Padé approximation and Laplace transform have been regarded as posttreatment tools that are used to obtain some results of nonlinear differential equations [8-14]. Gauss elimination, LU factorization, and chasing methods are classical direct methods [15], and Jacobi, Gauss-Seidel, SOR, (preconditioned) Krylov subspace, and (preconditioned) HSS methods are iteration methods for solving systems of linear equations [15-19]. Besides, Newton, Euler, Runge-Kutta, Newton-HSS, and Newton-Krylov subspace methods are numerical methods for solving systems of nonlinear equations [20-23]. In this paper, we deeply study $\mathrm{ADM}$ and the posttreatment technique for solving the model of HIV infection (1).

The type of HIV infection model (1) has been deeply discussed in $[3,4]$. Arafa et al. proposed generalized Euler method (GEM) to solve the fractional order model in [24]. Ongun used the LADM to solve the system of nonlinear differential equations (1) in [4]. Doğan proposed the multistep LADM to solve the system of nonlinear differential equations (1) in [3]. We will use ADM to solve HIV infection model (1), and we will utilize Padé approximation and Laplace transform for obtaining some new results.

The remainder of this paper is organized as follows. in Section 2, we apply ADM for solving HIV infection model (1). In Section 3, we use Padé approximation and Laplace transform as posttreatment technique to improve the results of ADM. In Section 4, we give numerical results for the system of nonlinear differential equations (1) and compare these results with those of the classical fourth-order RungeKutta (RK4) method.

\section{Application of ADM}

In this section, the classical ADM is used to solve the system of nonlinear differential equations (1), whose results are the same as that using the LADM [4]. For this class of problems, $\mathrm{ADM}$ is a simple and convenient method.

Now, let us use ADM to solve the system of nonlinear differential equations (1). Rewriting HIV infection model (1) in an operator form,

$$
\begin{aligned}
& L_{t} T=p-(\alpha-r) T-\frac{r}{T_{\max }} T^{2}-\frac{r}{T_{\max }} T I-k V T, \\
& L_{t} I=k V T-\beta I, \\
& L_{t} V=N \beta I-\gamma V,
\end{aligned}
$$

where the differential operator $L_{t}$ denotes

$$
L_{t}=\frac{\partial}{\partial t}
$$

Letting the inverse operator $L_{t}^{-1}$ be an integral operator of the form

$$
L_{t}^{-1}=\int_{0}^{t} d t
$$

and applying $L_{t}^{-1}$ on both sides of the equations in (3), we obtain

$$
\begin{aligned}
T= & T(0)+p t-(\alpha-r) L_{t}^{-1} T-\frac{r}{T_{\max }} L_{t}^{-1} T^{2} \\
& -\frac{r}{T_{\max }} L_{t}^{-1}(T I)-k L_{t}^{-1}(V T), \\
I= & I(0)+k L_{t}^{-1}(V T)-\beta L_{t}^{-1} I, \\
V= & V(0)+N \beta L_{t}^{-1} I-\gamma L_{t}^{-1} V .
\end{aligned}
$$

Using the ADM, we express the unknown items as the infinite series

$$
\begin{gathered}
T=\sum_{n=0}^{\infty} T_{n}, \\
I=\sum_{n=0}^{\infty} I_{n}, \\
V=\sum_{n=0}^{\infty} V_{n}
\end{gathered}
$$

and rewrite the nonlinear items as the Adomian polynomials

$$
\begin{gathered}
T^{2}=\sum_{n=0}^{\infty} A_{n}, \\
T I=\sum_{n=0}^{\infty} B_{n}, \\
V T=\sum_{n=0}^{\infty} C_{n} .
\end{gathered}
$$

Based on the above expressions and the initial conditions, we can obtain the following recursive relationship:

$$
\begin{aligned}
T_{0}= & r_{1}+p t \\
T_{n+1}= & -(\alpha-r) L_{t}^{-1} T_{n}-\frac{r}{T_{\max }} L_{t}^{-1} A_{n}-\frac{r}{T_{\max }} L_{t}^{-1} B_{n} \\
& -k L_{t}^{-1} C_{n}, \\
I_{0}= & r_{2}, \\
I_{n+1}= & k L_{t}^{-1} B_{n}-\beta L_{t}^{-1} I_{n}, \\
V_{0}= & r_{3}, \\
V_{n+1}= & N \beta L_{t}^{-1} I_{n}-\gamma L_{t}^{-1} V_{n},
\end{aligned}
$$

$$
(n=0,1,2, \ldots)
$$


By the aid of the software "Mathematica," we can write the first seven terms from the zeroth to the sixth components of $T(t), I(t)$, and $V(t)$ and then give their sums as follows:

$$
\begin{aligned}
& T_{q 6}:=\sum_{i=0}^{6} T_{i}(t) \\
& =0.1+0.397953 t+0.592849 t^{2}+0.588719 t^{3} \\
& +0.438295 t^{4}+0.260863 t^{5}+0.129195 t^{6} \\
& +0.0136354 t^{7}-0.278755 \times 10^{-4} t^{8}+1.00825 \\
& \times 10^{-8} t^{9}-1.34165 \times 10^{-12} t^{10}+8.06031 \\
& \times 10^{-17} t^{11}-2.22614 \times 10^{-21} t^{12}+2.29896 \\
& \times 10^{-26} t^{13} \\
& I_{q 6}:=\sum_{i=0}^{6} I_{i}(t) \\
& =2.7 \times 10^{-5} t+1.72737 \times 10^{-5} t^{2}-8.40515 \\
& \times 10^{-6} t^{3}+6.14728 \times 10^{-6} t^{4}-2.83586 \\
& \times 10^{-6} t^{5}+1.1533 \times 10^{-6} t^{6}-3.92561 \times 10^{-7} t^{7} \\
& +4.57012 \times 10^{-11} t^{8}-2.67794 \times 10^{-14} t^{9} \\
& +7.55134 \times 10^{-19} t^{10}+1.54535 \times 10^{-22} t^{11} \\
& -6.38153 \times 10^{-27} t^{12} \\
& V_{q 6}:=\sum_{i=0}^{6} V_{i}(t) \\
& =0.1-0.24 t+0.288041 t^{2}-0.230415 t^{3} \\
& +0.138243 t^{4}-0.0663528 t^{5}+0.0265397 t^{6} \\
& +2.48124 \times 10^{-6} t^{7}+1.28486 \times 10^{-10} t^{8} \\
& -3.58846 \times 10^{-15} t^{9}-1.44871 \times 10^{-19} t^{10} \\
& +2.57662 \times 10^{-23} t^{11}
\end{aligned}
$$

We now analyze the expressions $T_{q 6}, I_{q 6}$, and $V_{q 6}$. In fact, the first seven terms in the expressions of $T_{q 6}, I_{q 6}$, and $V_{q 6}$ are exact because the other terms will be changed by the remaining components. That is to say, the coefficients from $t^{0}$ to $t^{6}$ are invariable. So the truncated Taylor series of the approximate solutions $T_{q 6}, I_{q 6}$, and $V_{q 6}$ should be of the forms

$$
\begin{aligned}
& T_{t 6}(t)=0.1+0.397953 t+0.592849 t^{2}+0.588719 t^{3} \\
& +0.438295 t^{4}+0.260863 t^{5}+0.129195 t^{6}
\end{aligned}
$$

$$
\begin{gathered}
I_{t 6}(t)=10^{-5} \times\left(2.7 t+1.72737 t^{2}-0.840515 t^{3}\right. \\
\left.+0.614728 t^{4}-0.283586 t^{5}+0.11533 t^{6}\right), \\
V_{t 6}(t)=0.1-0.24 t+0.288041 t^{2}-0.230415 t^{3} \\
+0.138243 t^{4}-0.0663528 t^{5}+0.0265397 t^{6}
\end{gathered}
$$

Note that these results are the same as those of LADM in [4]. We believe that the results by using ADM are the same as those by using LADM, because LADM is essentially the same as that of ADM. LADM firstly uses the Laplace transform to the original equations. Then, based on the idea of ADM, a recursive relationship of the LADM is obtained [4]. And lastly, an approximate solution of the unknown function is derived by using the inverse Laplace transform. From the implementation procedure, we find that LADM is more complicated than ADM, because LADM uses not only Laplace transform but also inverse Laplace transform. However, ADM only uses inverse operator. So we can directly use ADM to solve the system of nonlinear differential equations (1).

\section{Posttreatment of ADM}

In this section, we first introduce Padé approximation and then combine Laplace transform with Padé approximation to conduct the results of ADM. By using this posttreatment technique, we can obtain better approximate solutions.

Assume that a function $f(x)$ has a Taylor expansion $f(x)=\sum_{i=0}^{\infty} c_{i} x^{i}$ at the zero point. We want to compute a Padé approximation to $f(x)$. To this end, let the $[L / M]$ Padé approximation have the form

$$
\left[\frac{L}{M}\right]=\frac{P_{L}(x)}{Q_{M}(x)}=\frac{a_{0}+a_{1} x+\cdots+a_{L} x^{L}}{1+b_{1} x+\cdots+b_{M} x^{M}} .
$$

By matching the coefficients of Taylor expansion of $f(x)$ with those of $[L / M]$ Padé approximation, we have

$$
\begin{aligned}
a_{0} & +a_{1} x+\cdots+a_{L} x^{L} \\
& =\left(1+b_{1} x+\cdots+b_{M} x^{M}\right)\left(c_{0}+c_{1} x+\cdots\right) .
\end{aligned}
$$

The coefficients $a_{0}, a_{1}, \ldots, a_{L}$ and $b_{1}, b_{2}, \ldots, b_{M}$ can be computed via the above equality. This means that the power series of $f(x)$ equals the $[L / M]$ Padé approximation from $x^{0}$ to $x^{L+M}$, or, in other words, it holds that

$$
f(x)-\frac{P_{L}(x)}{Q_{M}(x)}=O\left(x^{L+M+1}\right) .
$$

It is known that $[L / M]$ Padé approximation of a function is uniquely determined [25]. We want to apply Padé approximation to the approximate solutions of ADM. Because the coefficients of the first seven terms are invariable, to obtain better approximate solutions we should adopt [3/3] Padé approximation. In [4] the author was aware of the invariance 
of the coefficients of the first seven terms but used the [4/4] Padé approximation. According to the characteristic of the Padé approximation, the [3/3] Padé approximation should be better than the [4/4] Padé approximation [25].

By "Mathematica" software, the [3/3] Padé approximations of $T_{q 6}(t), I_{q 6}(t)$, and $V_{q 6}(t)$ are, respectively, given by

$$
\begin{aligned}
T_{P 3}= & \frac{0.1+0.249038 t+0.0889628 t^{2}+0.0369466 t^{3}}{1-1.48915 t+0.887248 t^{2}-0.220151 t^{3}}, \\
I_{P 3}= & 10^{-5} \\
& \times \frac{2.7 t+3.57914 t^{2}+0.50175 t^{3}}{1+0.685843 t+0.0583565 t^{2}-0.051507 t^{3}}, \\
V_{P 3}= & \frac{0.1-0.119945 t+0.0575645 t^{2}-0.011445 t^{3}}{1+1.20055 t+0.576566 t^{2}+0.115382 t^{3}}
\end{aligned}
$$

Usually, Laplace transform can make the coefficients of a power function become large, the constants disappear, and the index of unknown number increase by one. For example, applying Laplace transform to $T_{t 6}(t), I_{t 6}(t)$, and $V_{t 6}(t)$, we obtain

$$
\begin{aligned}
& \Psi\left(T_{t 6}(t)\right)=\frac{0.1}{s}+\frac{0.397953}{s^{2}}+\frac{1.1857}{s^{3}}+\frac{3.53231}{s^{4}} \\
& +\frac{10.5191}{s^{5}}+\frac{31.3036}{s^{6}}+\frac{93.0202}{s^{7}}, \\
& \Psi\left(I_{t 6}(t)\right)=10^{-5} \times\left(\frac{2.7}{s^{2}}+\frac{3.45473}{s^{3}}-\frac{5.04309}{s^{4}}\right. \\
& \left.+\frac{14.7535}{s^{5}}-\frac{34.0303}{s^{6}}+\frac{83.0376}{s^{7}}\right), \\
& \Psi\left(V_{t 6}(t)\right)=\frac{0.1}{s}-\frac{0.24}{s^{2}}+\frac{0.576081}{s^{3}}-\frac{1.38249}{s^{4}} \\
& +\frac{3.31783}{s^{5}}-\frac{7.96234}{s^{6}}+\frac{19.1086}{s^{7}} .
\end{aligned}
$$

For simplicity, we let $s=1 / t$. Then the above expressions become

$$
\begin{aligned}
& \Psi\left(T_{t 6}(t)\right)=0.1 t+0.397953 t^{2}+1.1857 t^{3} \\
& +3.53231 t^{4}+10.5191 t^{5}+31.3036 t^{6}+93.0202 t^{7}, \\
& \Psi\left(I_{t 6}(t)\right)=10^{-5} \times\left(2.7 t^{2}+3.45473 t^{3}-5.04309 t^{4}\right. \\
& \left.+14.7535 t^{5}-34.0303 t^{6}+83.0376 t^{7}\right), \\
& \Psi\left(V_{t 6}(t)\right)=0.1 t-0.24 t^{2}+0.576081 t^{3}-1.38249 t^{4} \\
& +3.31783 t^{5}-7.96234 t^{6}+19.1086 t^{7} .
\end{aligned}
$$

The highest indices of the Laplace transformations $\Psi\left(T_{t 6}(t)\right)$, $\Psi\left(I_{t 6}(t)\right)$, and $\Psi\left(V_{t 6}(t)\right)$ are 7 . Hence, Laplace transform increases the index by one for each component.

To combine Laplace transform and Padé approximation, we adopt [3/4] Padé approximation to approximate $\Psi\left(T_{t 6}(t)\right)$, $\Psi\left(I_{t 6}(t)\right)$, and $\Psi\left(V_{t 6}(t)\right)$. Because the highest indices of these power functions are 7, by Mathematica software again we have

$$
\begin{aligned}
& \Psi\left(T_{t 6}(t)\right)_{P} \\
& =\frac{0.1 t-0.521777 t^{2}-0.622784 t^{3}}{1.0-9.1973 t+18.5161 t^{2}+0.0436746 t^{3}-0.0324062 t^{4}}, \\
& \Psi\left(I_{t 6}(t)\right)_{P} \\
& =10^{-5} \\
& \times \frac{2.7 t^{2}+9.19088 t^{3}}{1.0+2.1245 t-0.850547 t^{2}-0.407785 t^{3}-0.071839 t^{4}}, \\
& \Psi\left(V_{t 6}(t)\right)_{P} \\
& =\frac{0.1 t-0.0321052 t^{2}-0.0160753 t^{3}}{1.0+2.07895 t-0.932089 t^{2}-0.388528 t^{3}+0.000113259 t^{4}} \\
& \Psi\left(T_{t 6}(t)\right)_{P} \\
& =\frac{0.1 s^{3}-0.521777 s^{2}-0.622784 s}{1.0 s^{4}-9.1973 s^{3}+18.5161 s^{2}+0.0436746 s-0.0324062}, \\
& \Psi\left(I_{t 6}(t)\right)_{P} \\
& =10^{-5} \\
& \times \frac{2.7 s^{2}+9.19088 s}{1.0 s^{4}+2.1245 s^{3}-0.850547 s^{2}-0.407785 s-0.071839}, \\
& \Psi\left(V_{t 6}(t)\right)_{P} \\
& =\frac{0.1 s^{3}-0.0321052 s^{2}-0.0160753 s}{1.0 s^{4}+2.07895 s^{3}-0.932089 s^{2}-0.388528 s+0.000113259} .
\end{aligned}
$$

Using the inverse Laplace transform, we have

$$
\begin{aligned}
& T_{\mathrm{LPL}}(t):=\Psi^{-1}\left(\Psi\left(T_{t 6}(t)\right)_{P}\right) \\
& =-0.0161448 e^{-0.0425685 t}-0.0174215 e^{0.0410798 t} \\
& +0.133575 e^{2.97986 t}-8.63837 \times 10^{-6} e^{6.21892 t}, \\
& I_{\mathrm{LPL}}(t):=\Psi^{-1}\left(\Psi\left(I_{t 6}(t)\right)_{P}\right)=10^{-5} \\
& \quad \times\left[0.420561 e^{-2.41214 t}+3.48737 e^{0.61872 t}\right. \\
& \quad-2 e^{-0.165538 t} \times(1.95397 \cos (0.143987 t) \\
& \quad-3.15941 \sin (0.143987 t))] \\
& \quad(t):=\Psi^{-1}\left(\Psi\left(V_{t 6}(t)\right)_{P}\right)=0.100023 e^{-2.39987 t} \\
& \quad-0.0000742602 e^{-0.272939 t} \\
& \quad+0.0000120428 e^{0.000291303 t} \\
& \quad+0.0000387879 e^{0.593571 t}
\end{aligned}
$$

where $\Psi^{-1}(\cdot)$ denotes the inverse Laplace transform. Till now, we have stated completely the posttreatment of ADM. In this way, we obtain the approximate solutions $T_{P 3}, I_{P 3}$, and $V_{P 3}$ by using the posttreatment Padé approximation and $T_{\mathrm{LPL}}(t), I_{\mathrm{LPL}}(t)$, and $V_{\mathrm{LPL}}(t)$ by using the posttreatment Padé approximation and Laplace transform, respectively. 


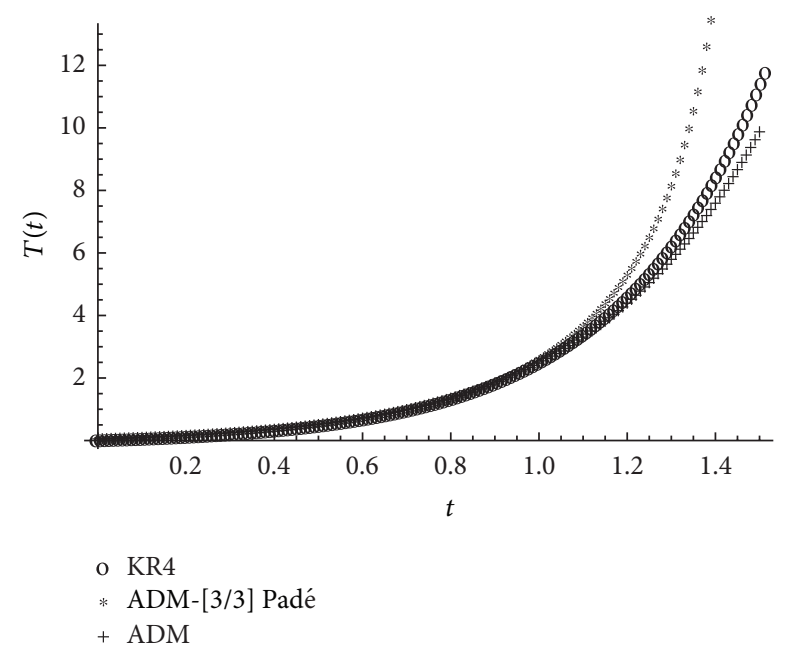

Figure 1: Curves of KR4, ADM-[3/3] Padé approximation, and ADM for $T(t)$.

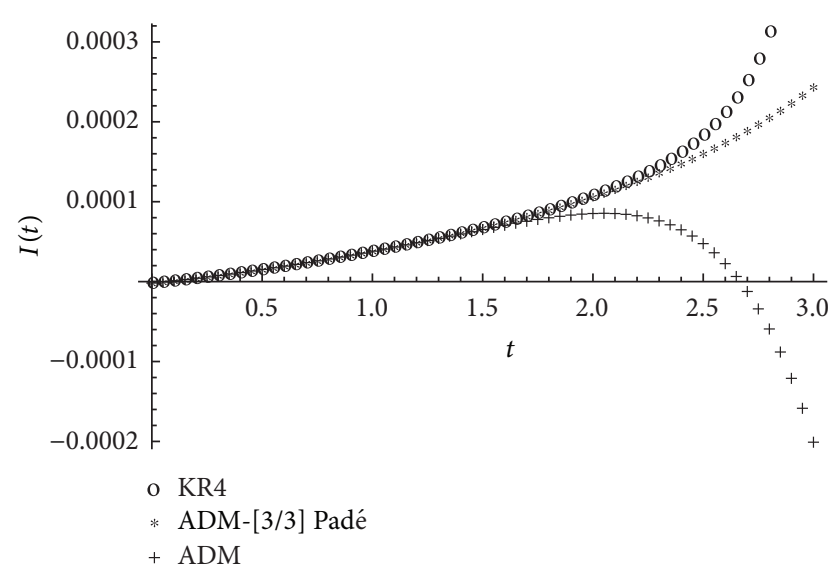

FIgure 2: Curves of KR4, ADM-[3/3] Padé approximation, and $\operatorname{ADM}$ for $I(t)$.

\section{Numerical Results}

In this section, we demonstrate the effectiveness of the posttreatment technique, that is, the Padé approximation and the Laplace transform. For simplicity, if the Pade approximation is used to conduct the results of ADM, we call it ADMPadé; if we use Padé approximation and Laplace transform to obtain the results of ADM, we call it ADM-LPL. We use the classical RK4 method, ADM, ADM-Padé, and ADM-LPL to solve HIV infection model (1). Numerical results of these methods are displayed by graphics.

Figures 1, 2, and 3 show the results of KR4, ADM-[3/3] Padé approximation, and ADM for functions $T(t), I(t)$, and $V(t)$, respectively. From Figure 1, we see that the result of $\mathrm{ADM}$ is closer to that of RK4 than that of ADM-[3/3] Padé approximation when the time is growing. From Figures 2 and 3 , we see that the results of ADM-[3/3] Padé approximation are closer to those of RK4 than those of ADM for $I(t)$ and $V(t)$ when the time is growing. From Figures 1, 2, and 3, we observe that Padé approximation is a better tool that can be

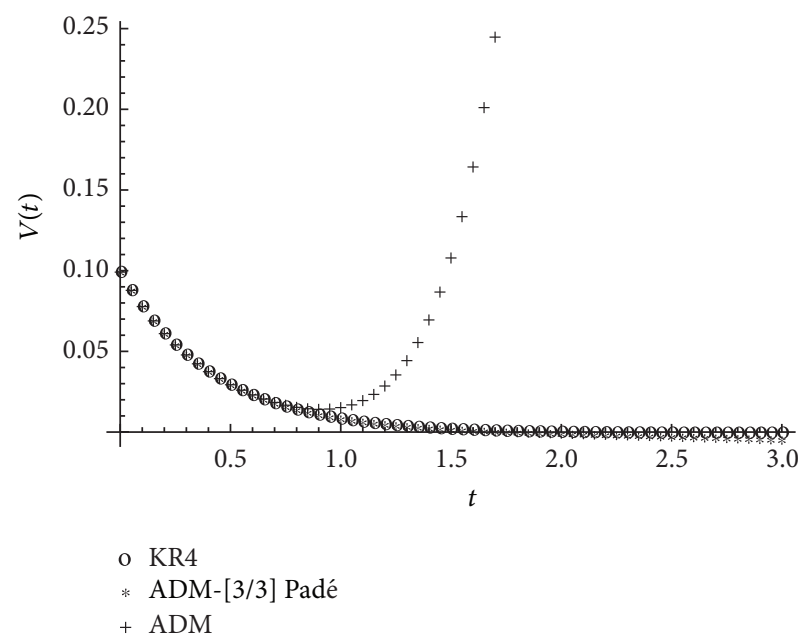

FIgUre 3: Curves of KR4, ADM-[3/3] Padé approximation, and ADM for $V(t)$.

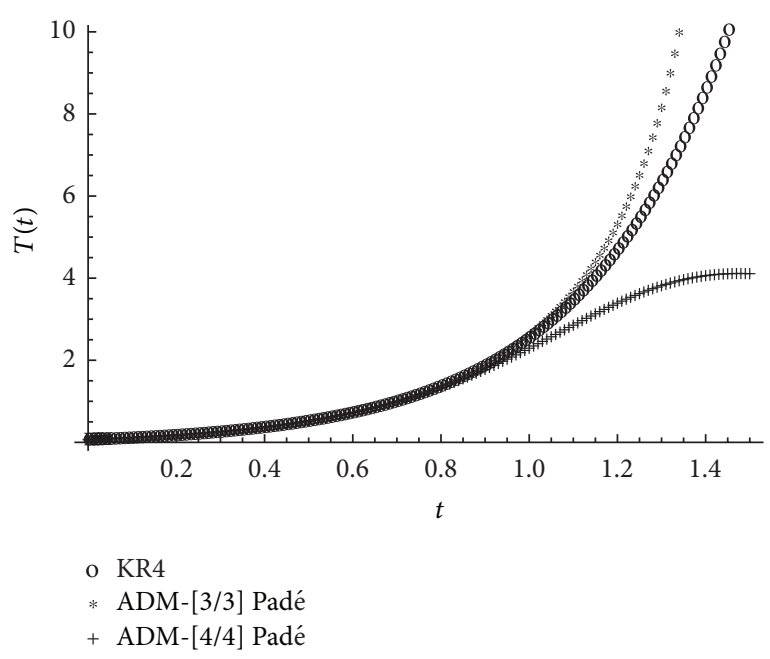

Figure 4: Curves of KR4 and ADM-[3/3] and ADM-[4/4] Padé approximations for $T(t)$.

used to obtain the results of ADM. This coincides with the theoretical analysis about Padé approximation. But, for HIV infection model (1), Figures 1, 2, and 3 show that the results of ADM-[3/3] Padé approximation are the same as those of $\mathrm{ADM}$ in a small time interval.

Figures 4, 5, and 6 show the results of KR4, ADM-[4/4] Padé approximation [4], and ADM-[3/3] Padé approximation for the functions $T(t), I(t)$, and $V(t)$, respectively. We see that the results of ADM-[3/3] Padé approximation are closer to those of KR4 than those of ADM-[4/4] Padé approximation when the time is growing, for functions $T(t), I(t)$, and $V(t)$ whatever. This coincides with the theoretical analysis in Section 3. Therefore, for HIV infection model (1), Figures 4, 5, and 6 show that the results of ADM-[3/3] Padé approximation are the same as those of ADM-[4/4] Padé approximation in a small time interval.

Figures 7, 8, and 9 show the results of KR4, ADM-[3/3] Padé approximation, and ADM-LPL for the functions $T(t)$, 


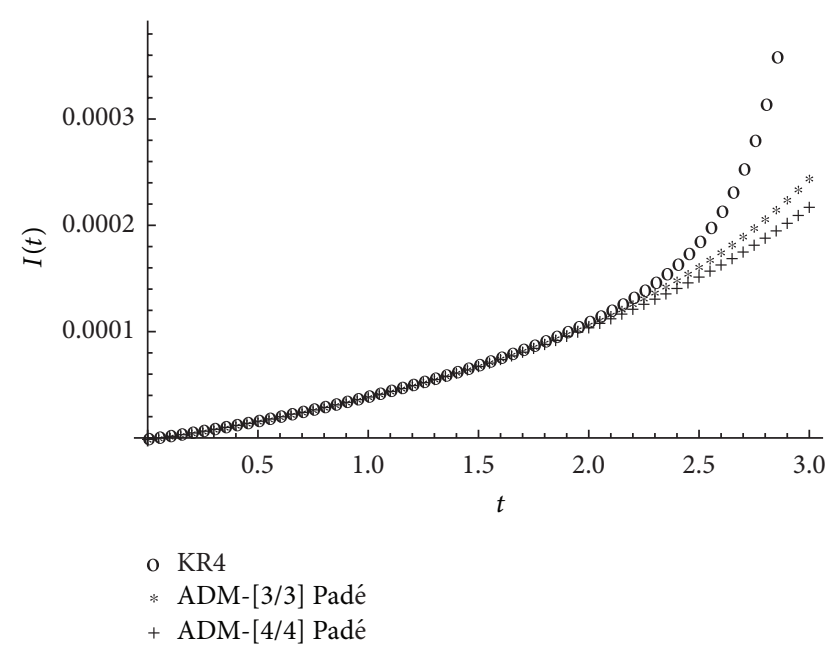

Figure 5: Curves of KR4 and ADM-[3/3] and ADM-[4/4] Padé approximations for $I(t)$.

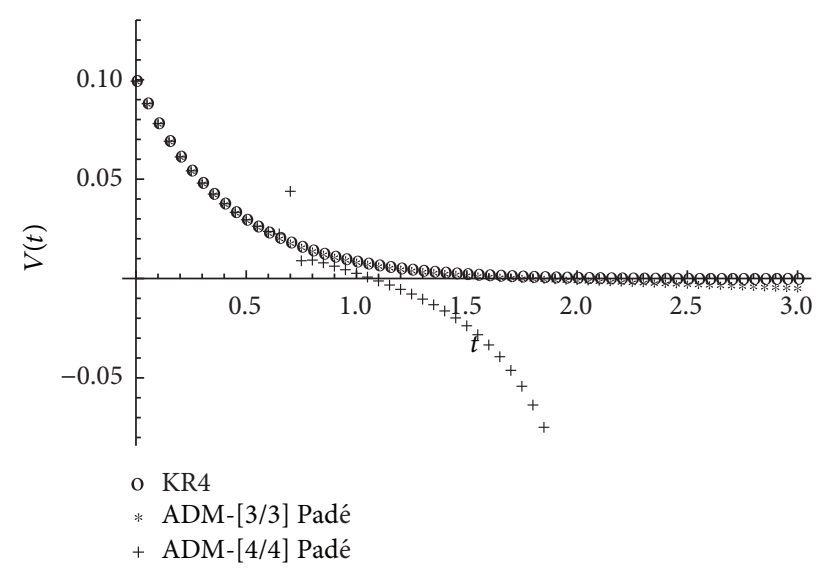

Figure 6: Curves of KR4 and ADM-[3/3] and ADM-[4/4] Padé approximations for $V(t)$.

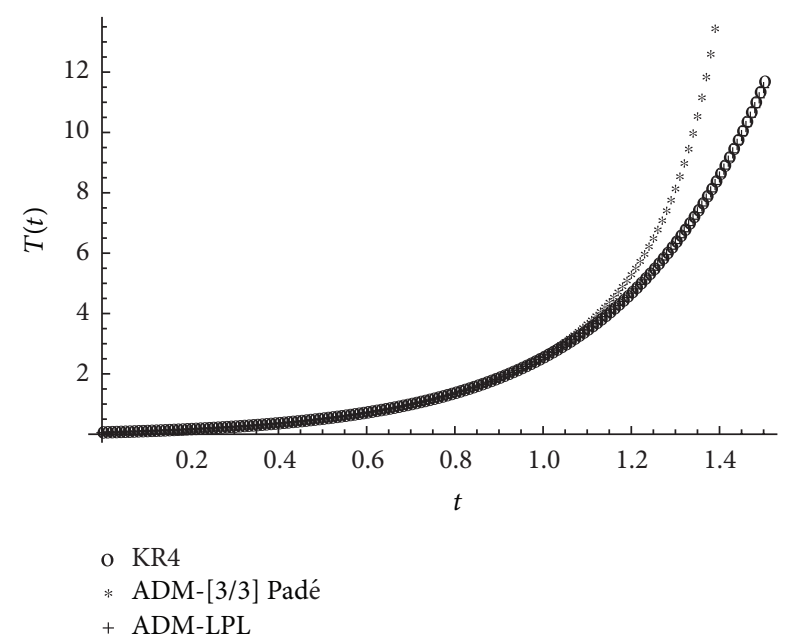

Figure 7: Curves of KR4, ADM-[3/3] Padé approximation, and ADM-LPL for $T(t)$.

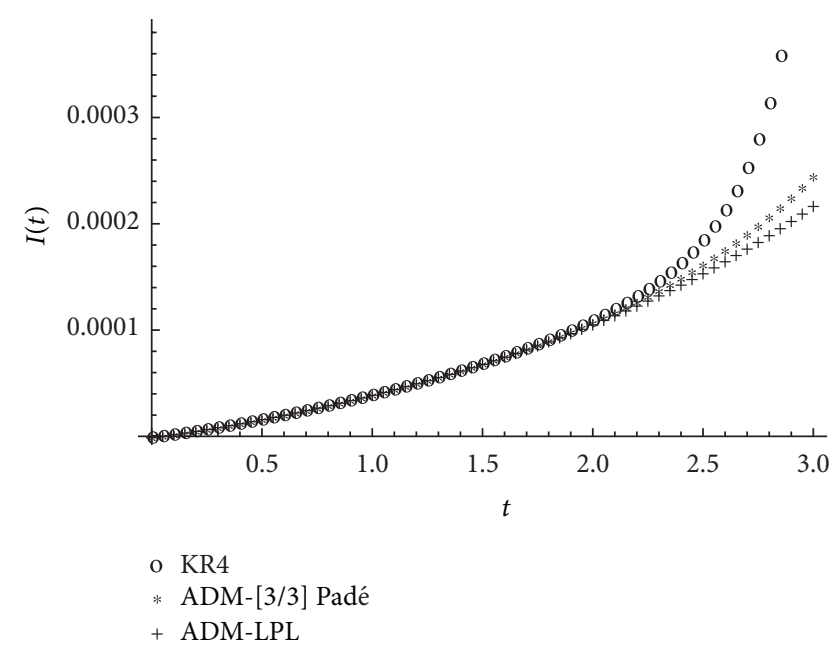

FIgURE 8: Curves of KR4, ADM-[3/3] Padé approximation, and ADM-LPL for $I(t)$.

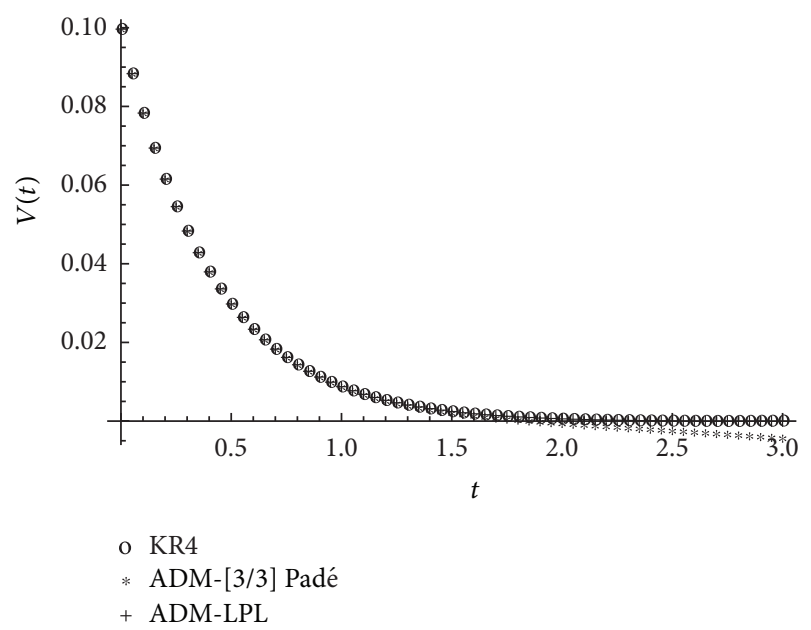

Figure 9: Curves of KR4, ADM-[3/3] Padé approximation, and ADM-LPL for $V(t)$.

$I(t)$, and $V(t)$, respectively. Figure 7 shows that the result of ADM-LPL is almost the same as that of RK4 for function $T(t)$. And Figure 8 shows that ADM-[3/3] Padé approximation outperforms ADM-LPL when the time is growing. Because of the scalar of the longitudinal coordinates in this figure, there is no much error for $I(t)$. Figure 9 shows that the result of ADM-LPL is almost the same as that of RK4 for the function $V(t)$, too. Therefore, for HIV infection model (1), Figures 7, 8, and 9 show that the results of ADM-[3/3] Padé approximation are the same as those of ADM-LPL in a small time interval.

In summary, the results of ADM, ADM-Padé, and ADMLPL are the same when they are used to solve HIV infection model (1) in a small time interval. From the results of these methods, we find that ADM-LPL is the best method for solving HIV infection model (1). Generally speaking, we need to find good posttreatment to conduct the result of ADM, which makes the results better when the time is growing. Padé approximation and Laplace transform are such good 
tools as needed. For HIV infection model (1), our numerical experiments have shown the effectiveness of posttreatment by using Padé approximation and Laplace transform.

\section{Conflict of Interests}

The authors declare that there is no conflict of interests regarding the publication of this paper.

\section{Acknowledgments}

This work is supported by the Natural Science Foundation of China (nos. 11372326, 11432015, and 11501038) and Beijing Outstanding Talents Foundation (no. 2014000020124G108).

\section{References}

[1] A. S. Perelson, "Modeling the interaction of the immune system with HIV," in Mathematical and Statistical Approaches to AIDS Epidemiology, C. Castillo-Chavez, Ed., vol. 83 of Lecture Notes in Biomathematics, pp. 350-370, Springer, New York, NY, USA, 1989.

[2] A. S. Perelson, D. E. Kirschner, and R. De Boer, "Dynamics of HIV infection of $\mathrm{CD}^{+}{ }^{+}$T cells," Mathematical Biosciences, vol. 114, no. 1, pp. 81-125, 1993.

[3] N. Doğan, "Numerical treatment of the model for HIV infection of $\mathrm{CD} 4^{+} \mathrm{T}$ cells by using multistep Laplace Adomian decomposition method," Discrete Dynamics in Nature and Society, vol. 2012, Article ID 976352, 11 pages, 2012.

[4] M. Y. Ongun, "The Laplace Adomian decomposition method for solving a model for HIV infection of $\mathrm{CD} 4^{+} \mathrm{T}$ cells," Mathematical and Computer Modelling, vol. 53, no. 5-6, pp. 597-603, 2011.

[5] G. Adomian, Stochastic Systems, Academic Press, New York, NY, USA, 1983.

[6] S. A. Khuri, "A Laplace decomposition algorithm applied to a class of nonlinear differential equations," Journal of Applied Mathematics, vol. 1, no. 4, pp. 141-155, 2001.

[7] J.-P. Sun, J.-C. Li, Q.-Q. Liu, and H.-Q. Zhang, "Approximate engineering solution for predicting groundwater table variation during reservoir drawdown on the basis of the boussinesq equation," Journal of Hydrologic Engineering, vol. 16, no. 10, pp. 791-797, 2011

[8] M. Dehghan, A. Hamidi, and M. Shakourifar, "The solution of coupled Burgers' equations using Adomian-Pade technique," Applied Mathematics and Computation, vol. 189, no. 2, pp. 10341047, 2007.

[9] Y.-C. Jiao, Y. Yamamoto, C. Dang, and Y. Hao, "An aftertreatment technique for improving the accuracy of Adomian's decomposition method," Computers and Mathematics with Applications, vol. 43, no. 6-7, pp. 783-798, 2002.

[10] T. R. Sivakumar and S. Baiju, "Shooting type Laplace-Adomian decomposition algorithm for nonlinear differential equations with boundary conditions at infinity," Applied Mathematics Letters, vol. 24, no. 10, pp. 1702-1708, 2011.

[11] A. M. Wazwaz, "The modified decomposition method and Padé approximants for a boundary layer equation in unbounded domain," Applied Mathematics and Computation, vol. 177, no. 2, pp. 737-744, 2006.
[12] A. M. Wazwaz, "Padé approximants and Adomian decomposition method for solving the Flierl-Petviashivili equation and its variants," Applied Mathematics and Computation, vol. 182, no. 2, pp. 1812-1818, 2006.

[13] A. M. Wazwaz, "The combined Laplace transform-Adomian decomposition method for handling nonlinear Volterra integro-differential equations," Applied Mathematics and Computation, vol. 216, no. 4, pp. 1304-1309, 2010.

[14] P. Yang, Y. Chen, and Z.-B. Li, "Adomian decomposition method and Padé approximants for solving the Blaszak-Marciniak lattice," Chinese Physics B, vol. 17, no. 11, pp. 3953-3964, 2008.

[15] G. H. Golub and C. F. Van Loan, Matrix Computations, The Johns Hopkins University Press, London, UK, 3rd edition, 1996.

[16] O. Axelsson and V. A. Barker, Finite Element Solution of Boundary Value Problems, Theory and Computation, Academic Press, New York, NY, USA, 1984.

[17] Z.-Z. Bai, G. H. Golub, and M. K. Ng, "Hermitian and skewHermitian splitting methods for non-Hermitian positive definite linear systems," SIAM Journal on Matrix Analysis and Applications, vol. 24, no. 3, pp. 603-626, 2003.

[18] D. Bertaccini, G. H. Golub, S. S. Capizzano, and C. T. Possio, "Preconditioned HSS methods for the solution of non-Hermitian positive definite linear systems and applications to the discrete convection-diffusion equation," Numerische Mathematik, vol. 99, no. 3, pp. 441-484, 2005.

[19] H. A. van der Vorst, Iterative Krylov Methods for Large Linear Systems, vol. 13 of Cambridge Monographs on Applied and Computational Mathematics, Cambridge University Press, Cambridge, UK, 2003.

[20] H.-B. An and Z.-Z. Bai, "A globally convergent Newton-GMRES method for large sparse systems of nonlinear equations," Applied Numerical Mathematics, vol. 57, no. 3, pp. 235-252, 2007.

[21] Z.-Z. Bai and X.-P. Guo, "On Newton-HSS methods for systems of nonlinear equations with positive-definite Jacobian matrices," Journal of Computational Mathematics, vol. 28, no. 2, pp. 235-260, 2010.

[22] Z.-Z. Bai and X. Yang, "On HSS-based iteration methods for weakly nonlinear systems," Applied Numerical Mathematics, vol. 59, no. 12, pp. 2923-2936, 2009.

[23] R. L. Burden, Numerical Analysis, Thomson Brooks/Cole, 2006.

[24] A. A. M. Arafa, S. Z. Rida, and M. Khalil, "Fractional modeling dynamics of HIV and $\mathrm{CD} 4{ }^{+}$T-cells during primary infection," Nonlinear Biomedical Physics, vol. 6, article 1, 2012.

[25] L. Wuytack, Padé Approximation and Its Applications, Springer, 1979. 


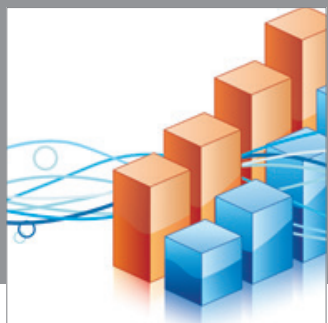

Advances in

Operations Research

mansans

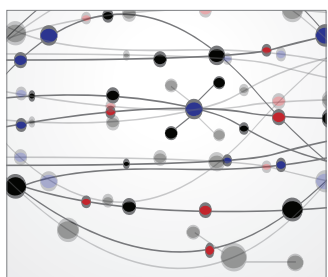

The Scientific World Journal
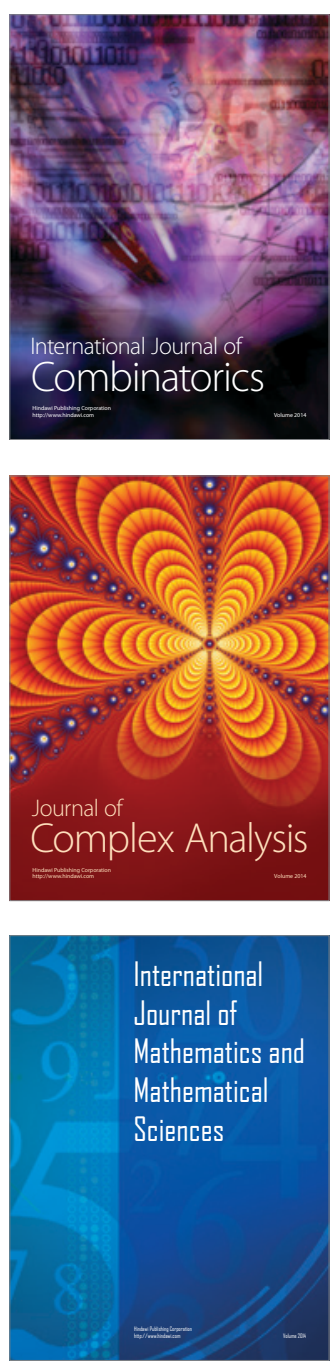
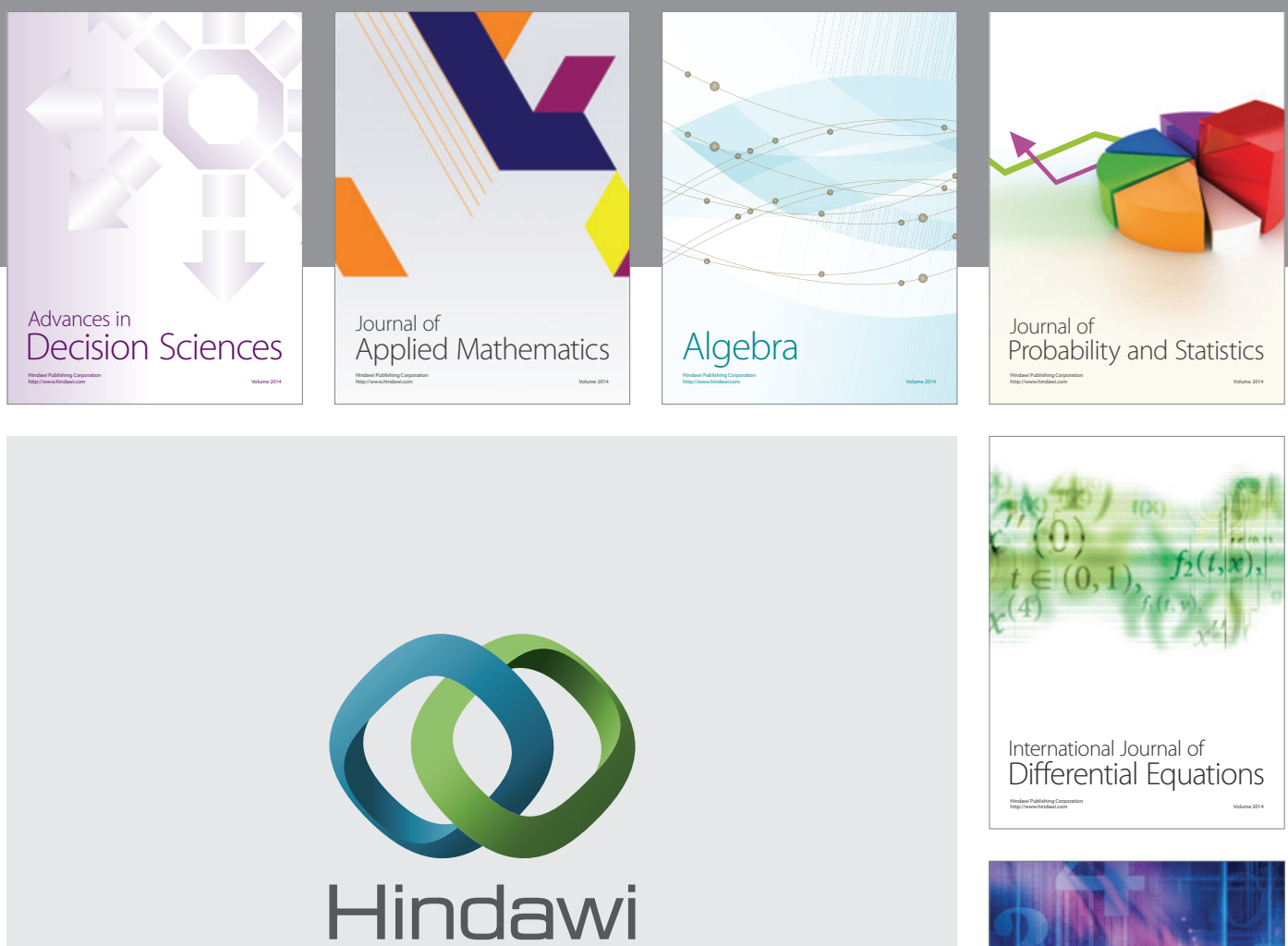

Submit your manuscripts at http://www.hindawi.com
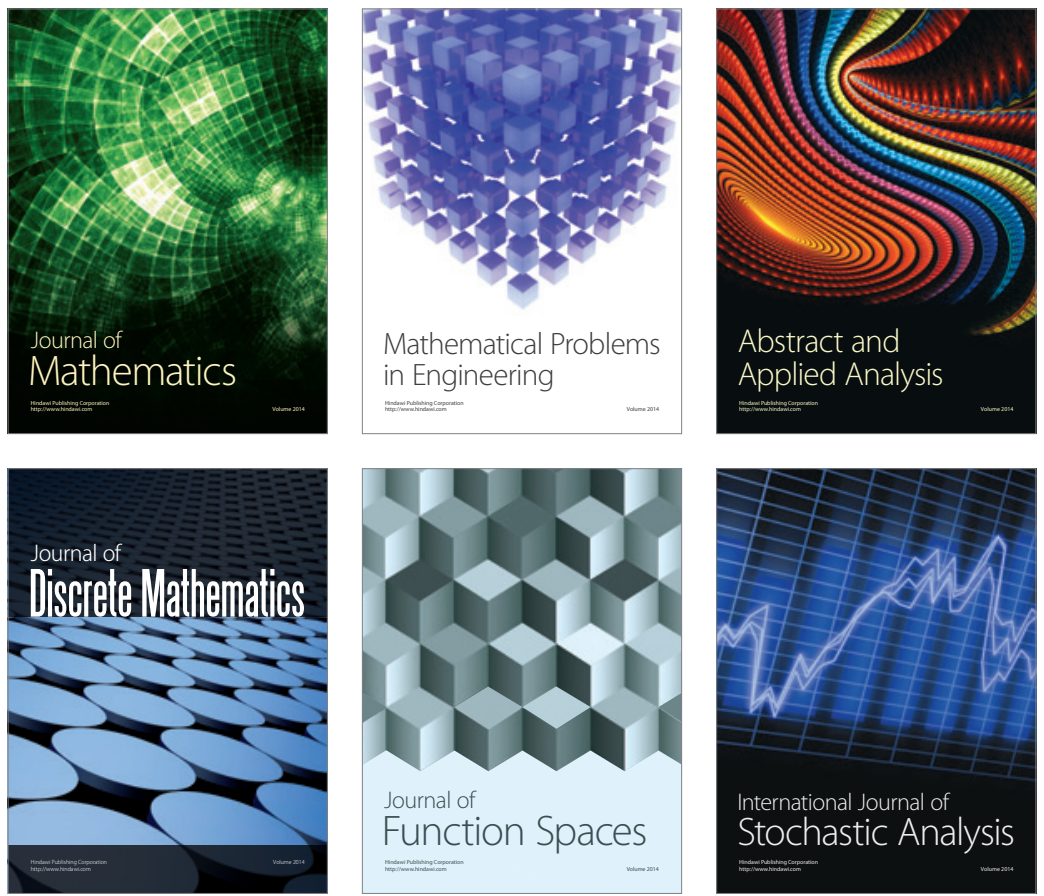

Journal of

Function Spaces

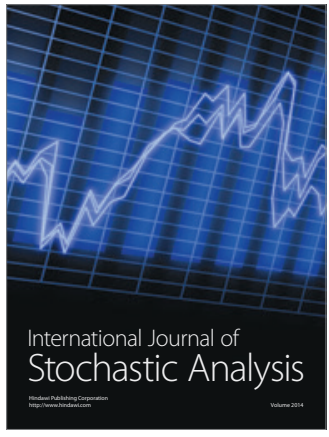

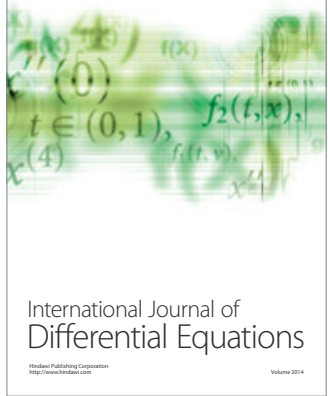
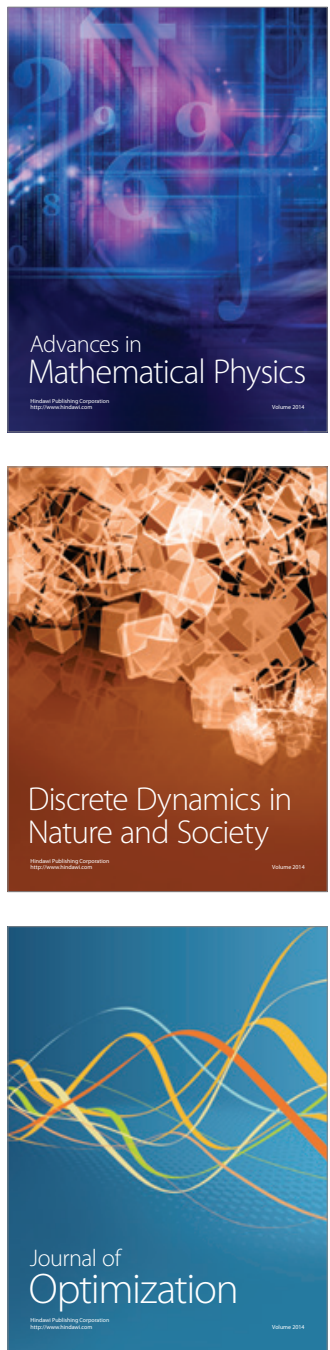